\title{
Revisiting the Optimal Linear Income Tax with Categorical Transfers
}

\author{
Sean Edward Slack ${ }^{1}$ \\ School of Economics \& Finance, University of St. Andrews, UK, KY16 9AR
}

\begin{abstract}
When individuals differ in both productivity and some categorical attribute, optimal linear/piecewise-linear tax expressions are written to capture cases where it is suboptimal to eliminate inequality in the average social marginal value of income between categorical groups. Simulations provide examples.

Keywords: Between-group Inequality; Categorical Benefit ; Linear Income Tax ; Social Marginal Value of Income

JEL Classification: H2; H53; I38
\end{abstract}

\section{Introduction}

When individuals differ in both their productivity and some categorical dimension such as disability, a well-established result is that categorical transfers should be set so as to eliminate inequality in the average social marginal value of income (smvi) between categorical groups (Diamond and Sheshinski, 1995; Parsons, 1996). The linear income tax framework has played an important role in the analysis of categorical transfers: proponents of flat tax schedules cite their administrative simplicity and enhanced work incentives; whilst analytically a flat tax captures the equity-efficiency tradeoff of income taxation more tractably than nonlinear taxation (Atkinson, 1995; Paulus and Peichl, 2009). ${ }^{2}$ The resulting optimal tax formulae are typically reported under the assumption that inequality in the average net smvi is in-

\footnotetext{
${ }^{1}$ E-mail address: ses34@st-andrews.ac.uk, Tel: + 44 (0) 1334468305

${ }^{2}$ Mirrlees (1971, p.208) discusses the desirability of approximately linear tax schedules.
} 
deed eliminated at the optimum (Viard, 2001). This assumption allows the optimal tax expression to be written as in the uni-dimensional model where individuals differ only in productivity: the numerator (equity considerations) is the negative of the covariance between earnings and the net smvi; whilst the denominator (efficiency considerations) captures the compensated labour supply response to a change in the net wage rate.

However, it is not immediately clear that this between-group inequality will always be eliminated at the optimum. Indeed, there may be cases where it is suboptimal to do so: if a sufficiently large fraction of the population are dependent on categorical transfers for consumption then the level of taxation required to equate the average net smvi of dependent and non-dependent groups may be too harmful to the latter group. This will also depend on the size of any revenue requirement for spending outside welfare.

Moreover, this is likely to hold beyond a simple flat tax framework. For example, progressive piecewise linear tax systems provide the government with additional tools to redistribute within categorical groups; but if shifting some of the tax burden away from lower earners in an able group: (i) pushes the average net smvi of that group further below that of a dependent group; and/or (ii) lowers tax revenue relative to the flat tax case, this may limit further the cases where it is optimal to eliminate between-group inequality.

This paper addresses this issue in both linear and piecewise linear income tax frameworks. It demonstrates that the optimal tax expressions can be written more generally to allow for cases where the average net smvi of categorical groups are not equated at the optimum. In these cases welfare provision is purely categorical, such that no universal benefit is provided. Alternatively, if between-group inequality is eliminated and there are resources left over a universal benefit is also provided. This reflects somewhat the ordering of priorities in real-world welfare systems: whilst most systems feature dimensions of both categorical and universal support, the former plays the prominent role. Extensive numerical simulations provide examples where between-group inequality is not eliminated at the optimum. Further, they indicate that it is more likely to arise under a progressive piecewise system for the reasons outlined above. 


\section{The Model}

\subsection{Background}

Individual preferences over consumption, $x \geq 0$, and leisure, $l \in[0,1]$, are represented by the utility function $u(x, l)$. The standard assumptions apply: $u$ is continuous; differentiable; increasing in both arguments $\left(u_{x}>0, u_{l}>0\right)$ and concave $\left(u_{x x}<0, u_{l l}<0, u_{x x} u_{l l}-u_{x l}^{2}>0\right)$; with both goods normal $\left(u_{l} u_{x x}-u_{x} u_{x l}<0\right)$.

For an individual with net wage $\omega \geq 0$ and unearned income $M \geq 0$, optimal labour supply $\left(H^{*}\right)$ and the resulting indirect utility function $(v)$ are defined by:

$$
\begin{aligned}
H^{*}(\omega, M) & \equiv \arg \max _{H \in(0,1)} u(\omega H+M, 1-H), \\
v(\omega, M) & \equiv u\left(\omega H^{*}+M, 1-H^{*}\right) .
\end{aligned}
$$

Let $\bar{\omega}(M)=u_{l}(M, 1) / u_{x}(M, 1)$ be the reservation wage satisfying: $H^{*}=$ $0 \forall \omega \leq \bar{\omega}$ and $H^{*}>0 \forall \omega>\bar{\omega}$; where $\bar{\omega}^{\prime}>0$. It follows that $\forall \omega<\bar{\omega}$ : $v(\omega, M)=u(M, 1)$ and thus $v_{M}(\omega, M)=u_{x}(M, 1)$. Contrastingly, Roy's identity $\left(v_{\omega}=v_{M} H^{*}\right)$ and the normality of leisure $\left(H_{M}^{*}<0\right)$ imply that $\forall \omega>\bar{\omega}: v_{\omega M}=v_{M M} H^{*}+v_{M} H_{M}^{*}<0$. So for $\omega>\bar{\omega}$ the marginal indirect utility of unearned income is strictly decreasing in the net wage rate.

\subsection{The Tax-Benefit System}

Consider a population of size 1 , where a fraction $\theta \in(0,1)$ of individuals face a zero quantity constraint on labour supply and are thus unable to work. Absent any form of state financial provision these individuals would have zero income to consume. The remaining $(1-\theta)$ individuals are able to work but differ in their underlying productivity $n \geq 0$, where $n$ is distributed with density function $f(n)$ and associated distribution function $F(n)$.

The government operates a tax-benefit system comprising: (i) a constant marginal income tax rate $t \in(0,1)$; (ii) a tax-free universal benefit $B \geq 0$ received unconditionally by all individuals in society; and (iii) a tax-free categorical benefit $C \geq 0$ that is perfectly targeted at unable individuals.

Let $y(n, 1-t, M) \equiv n H^{*}[n(1-t), M]$ be the gross earnings of a productivity $n$ individual; whilst $\bar{y}(1-t, M) \equiv \int y f(n) d n$ is the average gross earnings of able individuals. 
Under a strictly utilitarian criterion, social welfare is:

$$
W(t, B, C ; \theta)=\theta u(B+C, 1)+(1-\theta) \int v[n(1-t), B] f(n) d n
$$

The government's problem is thus described by:

$$
\begin{array}{ll} 
& \max _{t, B, C} W(t, B, C ; \theta) \\
\text { s.t. } & B+\theta C=(1-\theta) t \cdot \bar{y}(t, B)-R, \\
& t \in(0,1), B \geq 0, C \geq 0 .
\end{array}
$$

where $R \geq 0$ is an exogenous revenue requirement.

To discuss the results which follow, let the net smvi of a productivity $n$ individual be (Viard, 2001; Atkinson, 1995):

$$
s(n, t, M, \lambda)= \begin{cases}u_{x}(M, 1) & : n \leq \bar{n}(t, M) \\ v_{M}[n(1-t), M]+\lambda t y_{M}(n, 1-t, M) & : n>\bar{n}(t, M)\end{cases}
$$

where $\bar{n} \equiv \bar{\omega} /(1-t)$ and $\lambda$ is the shadow price of public expenditure. For working individuals $s$ captures - in welfare units - the fact that an increase in unearned income induces a worker to reduce their labour supply and, consequently, lowers tax revenue.

Letting $\hat{t}, \hat{B}$ and $\hat{C}$ be the optima resulting from (2), we have:

Result 1:

(i) $\hat{C}>0$ and $\hat{B} \geq 0$ satisfy:

$$
\bar{s}(\hat{t}, \hat{B}, \hat{\lambda}) \leq u_{x}(\hat{B}+\hat{C}, 1)=\hat{\lambda} ; \hat{B} \geq 0
$$

where the pair of inequalities holds with complementary slackness and $\hat{\lambda}$ is the shadow price of public expenditure at the optimum.

(ii) For $\beta \equiv(\lambda-\bar{s})$ and $r=y / \bar{y}$; $\hat{t}$ is implicitly characterised by:

$$
\frac{\hat{t}}{1-\hat{t}}= \begin{cases}\frac{\beta-\operatorname{Cov}(r, s)}{\hat{\lambda} \int r \mathcal{E}^{c} f(n) d n} & : \beta>0 \\ \frac{-\operatorname{Cov}(y, s)}{\hat{\lambda} \int y \mathcal{E}^{c} f(n) d n} & : \beta=0\end{cases}
$$

where $\mathcal{E}^{c}$ is the compensated elasticity of earnings with respect to the net of tax rate. 
Result 1(i) states that, budget allowing, $C$ should be set so as to eliminate inequality in the average net smvi between the unable and able subpopulations (see Viard, 2001). Further, so long as there is inequality in this dimension it is optimal to set $B=0$ because social welfare can be increased more through targeting resources at the unable.

Next, Result 1(ii) provides a general expression for the optimal linear tax rate that captures the equity (numerator) - efficiency (denominator) tradeoff inherent in income taxation. This differs from standard linear tax expressions whenever $\beta>0$, and thus whenever there is inequality in the average net smvi between the unable and able subpopulations. In this case, the numerator is composed of two terms. The first is $\beta$ itself, which will be larger the greater is the between-group disparity at the optimum. The second is the covariance between relative earnings $(r)$ and the net smvi. Notice that $\operatorname{Cov}(r, s) \cdot \bar{y}=\operatorname{Cov}(y, s)$, where the latter term is found in all linear tax formulae and captures a desire to redistribute from those of high productivity to those of lower productivity. ${ }^{3}$ The intuition for these two terms is that the presence of a dependent subpopulation shifts the equity focus away from disparities in earnings ability within the able subpopulation, and towards the between-group disparity in the average net smvi.

The denominator in (5) is unambiguously positive. Ceteris paribus, higher compensated elasticities of labour supply imply lower tax rates, with emphasis placed on both very high productivities and productivities at which the population is most dense.

\subsection{A Progressive Piecewise Linear Income Tax System}

Whilst a number of countries adopt a flat income tax, the majority employ progressive piecewise linear tax schedules (see Paulus and Peichl, 2009). This section motivates the idea that the above discussion is likely to hold in a more general setting. The next section then illustrates this numerically.

Let there be an earnings threshold $Y$ and two tax rates $t_{1} \leq t_{2}$ such that an individual's budget constraint is:

$$
x= \begin{cases}\left(1-t_{1}\right) y+B & : y \leq Y \\ \left(t_{2}-t_{1}\right) Y+\left(1-t_{2}\right) y+B & : y>Y\end{cases}
$$

The tax-benefit system remains otherwise as in Section 2.2.

\footnotetext{
${ }^{3}$ This covariance will be negative if: (i) $y_{n} \geq 0$; and (ii) $s_{n}<0$.
} 
To progress it is helpful to abstract from income effects ${ }^{4}$ through assuming preferences take the form:

$$
U(x, H)=u(x-g(H)) ; u^{\prime}>0, u^{\prime \prime}<0, g^{\prime}>0, g^{\prime \prime}>0
$$

As is well documented (Apps et al., 2014), there will be a 'bunching' of earnings at $Y$ for individuals who would earn more than $Y$ if additional earnings were still taxed at rate $t_{1}$; but choose not to because they are in fact taxed at rate $t_{2}$. Formally, this bunching occurs for $n \in(\tilde{n}, \tilde{\tilde{n}}]$; where:

$$
\tilde{n}\left(1-t_{1}\right) \equiv g^{\prime}(Y / \tilde{n}) \quad, \quad \tilde{\tilde{n}}\left(1-t_{2}\right) \equiv g^{\prime}(Y / \tilde{\tilde{n}})
$$

and for $n \in(\tilde{n}, \tilde{\tilde{n}}):\left(1-t_{1}\right)>g^{\prime}(Y / n)>\left(1-t_{2}\right)$.

Individuals with $n \in(0, \tilde{n}]$ choose $y \leq Y$ so that $n\left(1-t_{1}\right)=g^{\prime}(y / n)$; whilst individuals with $n \in(\tilde{\tilde{n}}, \infty)$ choose $y>Y$ so that $n\left(1-t_{2}\right)=g^{\prime}(y / n)$.

If we denote by $v\left(n, t_{1}, t_{2}, Y, M\right)$ the indirect utility resulting from the optimal choice of $y$ we can establish that:

$$
\frac{\partial v}{\partial t_{1}}=\left\{\begin{array}{ll}
-v_{M} y & : n \in(0, \tilde{n}] \\
-v_{M} Y & : n \in(\tilde{n}, \infty)
\end{array} \quad ; \frac{\partial v}{\partial t_{2}}= \begin{cases}0 & : n \in(0, \tilde{\tilde{n}}] \\
-v_{M} \cdot(y-Y) & : n \in(\tilde{\tilde{n}}, \infty)\end{cases}\right.
$$

and:

$$
\frac{\partial v}{\partial Y}= \begin{cases}0 & : n \in(0, \tilde{n}] \\ v_{M} \cdot\left(1-t_{1}\right)-g^{\prime}(Y / n) / n>0 & : n \in(\tilde{n}, \tilde{\tilde{n}}] \\ v_{M} \cdot\left(t_{2}-t_{1}\right)>0 & : n \in(\tilde{\tilde{n}}, \infty)\end{cases}
$$

As we are abstracting from income effects, an individual's smvi is $s=v_{M}$ and once more $\bar{s}=\int s f(n) d n$.

The government's optimisation problem is now:

$$
\max _{t_{1}, t_{2}, Y, B, C} W=\theta u(B+C)+(1-\theta) \int v\left(n, t_{1}, t_{2}, Y, B\right) f(n) d n
$$

s.t. $B+\theta C$

$$
\begin{aligned}
& =(1-\theta)\left\{t_{1}\left\langle\int^{\tilde{n}} y f(n) d n+Y[1-F(\tilde{n})]\right\rangle+t_{2} \int_{\tilde{\tilde{n}}}(y-Y) f(n) d n\right\}-R \\
& t_{1} \in(0,1), t_{2} \in(0,1), t_{1} \leq t_{2}, Y \geq 0, B \geq 0, C \geq 0
\end{aligned}
$$

\footnotetext{
${ }^{4}$ This ensures that the f.o.c. for earnings $y>Y$ is unaffected by $t_{1}$, which simplifies the analysis (see Apps et al., 2014).
} 
Letting $\hat{t}_{1}, \hat{t}_{2}, \hat{Y}, \hat{B}$ and $\hat{C}$ denote the resulting optima, we have:

\section{Result 2:}

(i) $\hat{C}>0$ and $\hat{B} \geq 0$ satisfy:

$$
\bar{s}\left(\hat{t}_{1}, \hat{t}_{2}, \hat{Y}, \hat{B}\right) \leq u^{\prime}(\hat{B}+\hat{C})=\hat{\lambda}
$$

(ii) For $\mathcal{E}_{i}=\frac{\left(1-t_{i}\right)}{y} \frac{\partial y}{\partial\left(1-t_{i}\right)} ; i \in\{1,2\}, \hat{t}_{1}, \hat{t}_{2}$ and $\hat{Y}$ are characterised by:

$$
\begin{aligned}
\left(t_{1}\right): & \frac{\hat{t}_{1}}{1-\hat{t}_{1}}=\frac{\beta \hat{Y}+\int^{\tilde{n}}(Y-y)(s-\hat{\lambda}) f(n) d n}{\hat{\lambda} \int^{\tilde{n}} y \mathcal{E}_{1} f(n) d n} \\
\left(t_{2}\right): & \frac{\hat{t}_{2}}{1-\hat{t}_{2}}=\frac{\int_{\tilde{n}}(y-\hat{Y})(\hat{\lambda}-s) f(n) d n}{\hat{\lambda} \int_{\tilde{\tilde{n}}} y \mathcal{E}_{2} f(n) d n} \\
(Y): & \int_{\tilde{n}}^{\tilde{\tilde{n}}}\left(\frac{\partial v}{\partial Y}+\hat{\lambda} \hat{t}_{1}\right) f(n) d n=\left(\hat{t}_{2}-\hat{t}_{1}\right) \int_{\tilde{\tilde{n}}}(\hat{\lambda}-s) f(n) d n
\end{aligned}
$$

Result 2(i) parallels Result 1(i): a universal benefit will only be provided conditional on categorical transfers eliminating inequality in the average smvi. Result 2(ii) characterises the optimal tax parameters and is analogous to Apps et al. (2014). The important difference with these authors is that the presence of a dependent population changes how we write the expression for $\hat{t}_{1}$. In particular, whilst both the expressions for $\hat{t}_{1}$ and $\hat{t}_{2}$ have equity concerns in the numerator and efficiency concerns in the denominator, it is only the numerator of the former than contains $\beta$; entering as $\beta Y$. The intuition is seemingly that an increase in $t_{1}$ has no distortionary effect on the gross earnings of those with $n \in(\tilde{n}, \infty)$ and is therefore an effective tool to help reduce $\beta$. This does, of course, come at the cost of imposing a higher tax rate on those with $n \in(0, \tilde{n})$ and thus those with lower productivities. Finally, (12) illustrates that $Y$ should be set so as to equate (i) the marginal benefit of allowing individuals with $n \in(\tilde{n}, \tilde{\tilde{n}})$ to work more at rate $t_{1}$ (and in welfare units the associated increase in tax revenue); with (ii) the marginal cost of foregone tax revenue from those with $n \in(\tilde{\tilde{n}}, \infty)$, weighted by the positive term $\int_{\tilde{\tilde{n}}}(\lambda-s) f(n) d n$.

\section{Numerical Simulations}

The optimal tax expressions in Section 2 are endogenous to the tax rate, thus rendering comparative statics predictions scarce. To provide examples 
where $\beta>0$ at the optimum, we therefore turn to numerical methods.

Following Saez (2001) and Atkinson (1995) let preferences be of the isoelastic form:

$$
u(x, H)=\log \left(x-\alpha \frac{H^{1+k}}{1+k}\right)
$$

where in this setting $1 / k$ is the constant elasticity of labour supply with respect to the net wage rate and $\alpha$ is a constant. Low labour elasticities are observed empirically and we follow convention by setting $1 / k=0.25$.

Productivities are Pareto distributed where $f(n)=\mu \underline{n}^{\mu} / n^{\mu+1} \forall n \geq \underline{n}$. The Pareto distribution captures well the upper tail of observed income distributions and its adoption in the more recent optimal tax literature has supported increasing marginal tax rates on higher earners. ${ }^{5}$ It would therefore seem appropriate for simulating progressive piecewise tax schedules. To capture how the spread of abilities affects the results, we consider two alternative distributions: (i) $\underline{n}=1, \mu=4$; and (ii) $\underline{n}=1.067, \mu=5$, where $\underline{n}$ is adjusted so that the average productivity is 1.333 in both cases. The second distribution has a smaller spread of abilities than the first.

The remaining parameter choices are $\alpha=8 ; \theta \in\{0.10,0.15\}$ and $R \in$ $\{0,0.10,0.15,0.20\}$. The leisure preference parameter $\alpha$ is set so that the average worker works roughly $2 / 3$ of their time endowment. The values of the unable subpopulation size $\theta$ seem sensible following statistics on realworld welfare programs (Mcinnes, 2012). Finally, $R=0.2$ corresponds to $17 \%$ of maximum GDP (average productivity) when $\theta=0.1$.

Table 1 displays the numerical results and its structure is described in the caption immediately below the table. The most immediate observation is that there are indeed cases under both the flat tax and piecewise linear tax schedules where $\beta>0$ at the optimum. The existence of a critical flat $\operatorname{tax} t^{*}$ that generates just enough tax revenue to set $\beta=0$ illustrates that it is certainly possible to eliminate inequality in the average smvi; but this option may not be exercised because such a tax rate hurts able individuals too much. Moreover, there are a number of cases where $\beta=0$ under the flat tax schedule, but $\beta>0$ under the corresponding piecewise schedule. The intuition is clear: under the piecewise system the government has additional

\footnotetext{
${ }^{5}$ Extensive numerical results with alternative functional forms (e.g. CES) and distributions (e.g. LogNormal) provide further examples where $\beta>0$ and are available from the author upon request. Python 2.7 was used for the numerical analysis.
} 


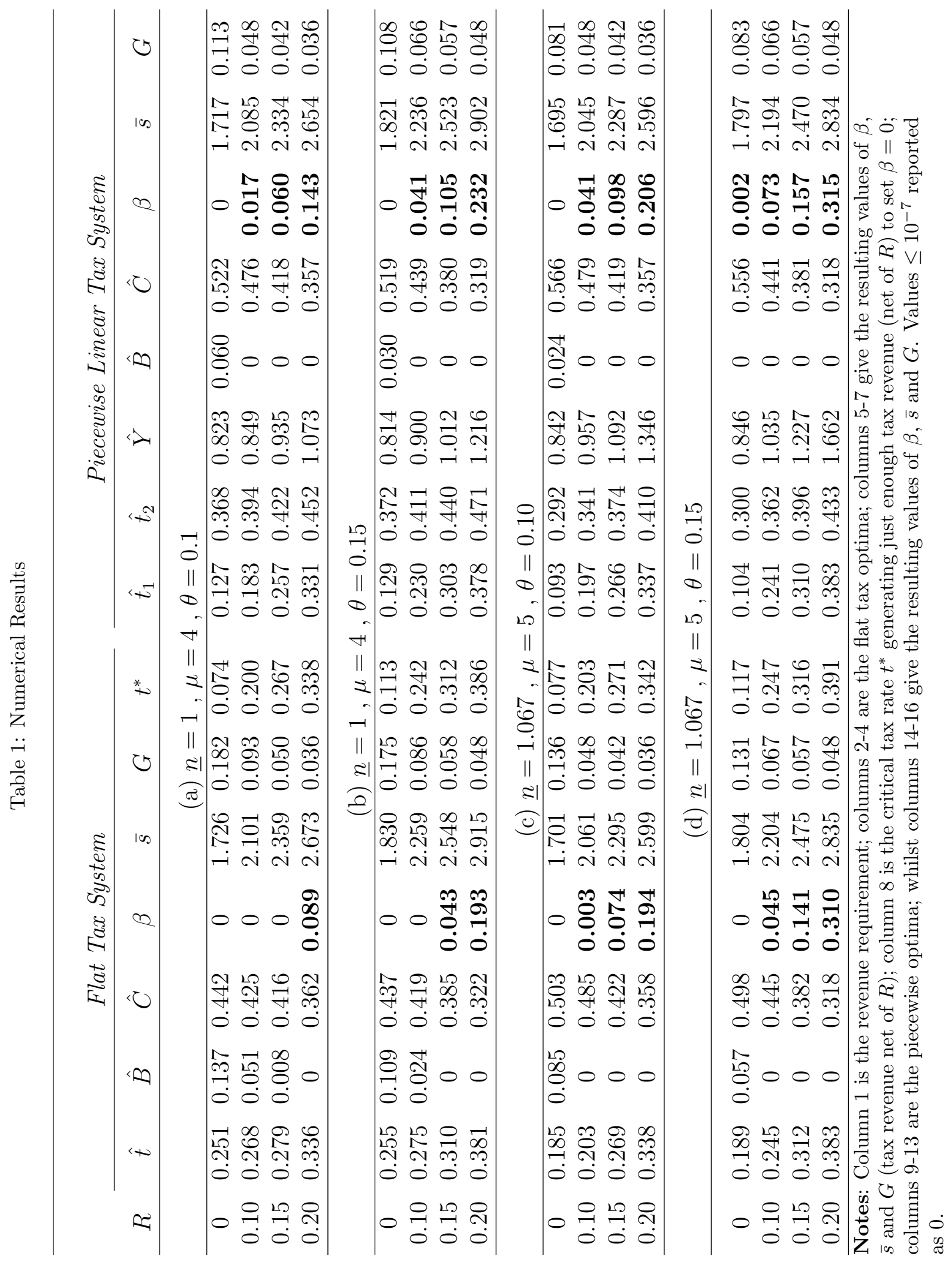


tools to affect the distribution of earnings within the able subpopulation. Setting $t_{1}<t_{2}$ shifts some of the tax burden away from lower productivity individuals and towards higher productivity individuals, thus lowering $\bar{s}$ relative to that under the flat tax optimum. Even though the unable receive a similar benefit income to the flat tax case, $\beta$ is necessarily higher due to the fall in $\bar{s}$. In these cases net tax revenue is also lower under the piecewise schedule. Note that where $\beta>0$ under both types of system, the unable tend to receive a slightly lower level of categorical support under the piecewise system than they do under the flat tax system. Finally, given that the flat tax is always available but not chosen, welfare is higher under the piecewise system (to save on space this is omitted from the table).

Table 1 illustrates that an increase in $\theta$ or $R$ increases the number of cases where $\beta>0$. Interestingly, a mean-preserving reduction in the spread of abilities increases: (i) the number of cases where $\beta>0$; and (ii) the magnitude of $\beta$ for cases where $\beta>0$ already. This arises because, for any given value of $t$, both $\bar{s}$ and tax revenue fall. Consequently, the critical tax rate $t^{*}$ at which between-group inequality is eliminated rises. ${ }^{6}$ Figure 1 summarizes the conditions where $\beta>0$ in $(R, \theta)$ space.

Example with Income Effects. The numerical examples where $\beta>0$ continue to hold if we introduce income effects. Let preferences be (Saez, 2001):

$$
u(x, H)=\log (x)-\alpha \log \left(1+\frac{H^{1+k}}{1+k}\right)
$$

where $\alpha=8$ and $1 / k=0.25$. Table 2 presents results for the flat tax case.

[FIGURE 1 HERE (scale=0.45), Caption: Between-Group Inequality at the Optimum in $(R, \theta)$ Space ]

\section{Concluding Remarks}

To summarise, the analysis of categorical transfers in linear/piecewise linear income tax frameworks generates optimal tax expressions that depend on whether or not categorical transfers eliminate inequality in the average smvi across categorical groups. Numerical simulations provide examples where it is suboptimal to finance categorical transfers up to this point: such as when

\footnotetext{
${ }^{6}$ Note that $t^{*}$ would still rise even if we $\operatorname{did} \operatorname{not}$ adjust $\underline{n}$ so as to keep mean productivity constant (for any given $t: \bar{s}$ may increase but tax revenue falls).
} 
Table 2: Flat Tax Optima with Income Effects

\begin{tabular}{ccccccc}
\hline$R$ & $\hat{t}$ & $\hat{B}$ & $\hat{C}$ & $\beta$ & $\bar{s}$ & $t^{*}$ \\
\hline \hline \multicolumn{7}{c}{ (a) $\underline{n}=1, \mu=4, \theta=0.15$} \\
\hline 0 & 0.235 & 0.073 & 0.662 & 0 & 1.360 & 0.146 \\
0.10 & 0.263 & 0 & 0.652 & $\mathbf{0 . 0 1 0}$ & 1.525 & 0.264 \\
0.20 & 0.376 & 0 & 0.552 & $\mathbf{0 . 1 0 3}$ & 1.710 & 0.381 \\
\hline \multicolumn{7}{c}{ (b) $\underline{n}=1.067, \mu=5, \theta=0.15$} \\
\hline 0 & 0.167 & 0.015 & 0.733 & 0 & 1.336 & 0.149 \\
0.10 & 0.263 & 0 & 0.651 & $\mathbf{0 . 0 4 8}$ & 1.487 & 0.267 \\
0.20 & 0.376 & 0 & 0.551 & $\mathbf{0 . 1 4 9}$ & 1.665 & 0.384 \\
\hline
\end{tabular}

there is a sufficiently large dependent population and/or the government has spending commitments outside welfare. How these optimal tax expressions change when categorical transfers are administered with classification errors - and thus where it may be impossible to eliminate inequality in the average smvi - warrants investigation.

\section{Acknowledgements}

Financial support from the AXA Research Fund is gratefully acknowledged. I also thank the anonymous referee for their helpful comments and suggestions.

\section{References}

Apps, P., Long, N. V., Rees, R., 2014. Optimal Piecewise Linear Income Taxation. Journal of Public Economic Theory 16 (4), 523-545.

Atkinson, A. B., Oct. 1995. Public Economics in Action: The Basic Income/ Flat Tax Proposal. Oxford University Press, Oxford.

Diamond, P., Sheshinski, E., 1995. Economic aspects of optimal disability benefits. Journal of Public Economics 57, 1-23.

Mcinnes, R., 2012. ESA and incapacity benefit statistics. Tech. rep., House of Commons Library. 
Mirrlees, J., 1971. An Exploration in the Theory of Optimal Income Taxation. The review of economic studies 38 (2), 175-208.

Parsons, D., 1996. Imperfect 'tagging'in social insurance programs. Journal of Public Economics 62, 183-207.

Paulus, A., Peichl, A., 2009. Effects of flat tax reforms in Western Europe. Journal of Policy Modeling 31, 620-636.

Saez, E., 2001. Using Elasticities to Derive Optimal Income Tax Rates. Review of Economic Studies 68, 205-229.

Viard, A., 2001. Optimal Categorical Transfer Payments: The Welfare Economics of Limited LumpSum Redistribution. Journal of Public Economic Theory 3 (December 2000), 483-500. 